01

\title{
Является ли кинковое решение нелинейного уравнения Клейна-Гордона солитоном?
}

\author{
(С) Д.В. Завьялов, ${ }^{1}$ В.И. Конченков, ${ }^{1}$ С.В. Крючков ${ }^{1,2}$ \\ ${ }^{1}$ Волгоградский государственный технический университет, \\ 400005 Волгоград, Россия \\ ${ }^{2}$ Волгоградский государственный социально-педагогический университет, \\ 400066 Волгоград, Россия \\ e-mail: svkruchkov@yandex.ru
}

Поступило в Редакцию 25 октября 2018 г.

В окончательной редакции 25 октября 2018 г.

Принято к публикации 29 апреля 2019 г.

Численно исследована возможность существования солитонного решения обобщенного уравнения синусГордона, известного так же как уравнение Крючкова-Кухаря. Это уравнение описывает распространение электромагнитных волн в сверхрешетке на основе графена. Оценены вычислительные ошибки, связанные с тем, что кинковое решение рассматриваемого уравнения задано неявно, а также исследовано изменение формы кинков, движущихся навстречу друг другу, до и после столкновения. На основе полученных результатов сделан вывод о том, что рассматриваемое кинковое решение не является солитоном.

Ключевые слова: солитонное решение, уравнение Крючкова-Кухаря, коэффициент корреляции.

DOI: $10.21883 / J T F .2019 .10 .48160 .379-18$

\section{Введение}

Исследованию условий возникновения, распространения, затухания нелинейных волн (в том числе уединенных волн) в низкоразмерных материалах посвящен ряд работ (например, [1-5]). Интерес к таким волнам продиктован в том числе возможностью использования уединенных волн в устройствах кратковременного хранения информации и в устройствах связи. В частности, в оптоэлектронике широко изучаются плазмонполяритонные волны, распространяющиеся на границе металла и изолятора, ведутся исследования распространения уединенных волн в волноводах на основе графена [6-8]. Наиболее подробно изучены уединенные волны в полупроводниковых сверхрешетках (например, [5,9]), где эволюция векторного потенциала волны, распространяющейся вдоль слоев структуры, описывается уравнением синус-Гордона (SGE). Это уравнение детально исследовано и описано во многих монографиях.

После открытия графена внимание исследователей сосредоточивается на наноструктурах на его основе, в частности, на графеновых сверхрешетках (ГСР). С одной стороны, в таких системах проявляются свойства, характерные для графена, например, взаимная зависимость движений носителей тока в перпендикулярных друг другу направлениях. С другой стороны, наличие дополнительного периодического потенциала, имеющего период, существенно больший, чем период основной решетки, приводит к возможности проявления нелинейных свойств, характерных для сверхрешеток. Среди таких явлений - эффекты, связанные с распространением уединенных электромагнитных волн. В работе [10] исследована возможность существования уединенных электромагнитных волн в графеновой сверхрешетке, выведено уравнение, описывающее распространение таких волн в рассматриваемой структуре. В литературе это уравнение получило название уравнение КрючковаКухаря (KKeq) [11]. Это уравнение является обобщением уравнения синус-Гордона, в явном виде учитывающим взаимную зависимость движений носителей тока в перпендикулярных друг другу направлениях. С использованием рассматриваемого уравнения исследован эффект фотонного увлечения носителей тока уединенными электромагнитными волнами в графеновой сверхрешетке [12,13], исследовано влияние высокочастотного электромагнитного излучения на распространение коротких электромагнитных импульсов в ГСР, изучена возможность поддержания формы уединенной волны под действием внешнего высокочастотного электрического поля лазера $[14,15]$.

\section{Постановка задачи}

В безразмерном виде уравнение распространения электромагнитной (ЭМ) волны в ГСР имеет вид [10]

$$
\frac{\partial^{2} \varphi}{\partial \tau^{2}}-\frac{\partial^{2} \varphi}{\partial s^{2}}+\frac{b^{2} \sin \varphi}{\sqrt{1+b^{2}(1-\cos \varphi)}}=0 .
$$

Здесь $\tau=t \omega_{0}-$ безразмерное время, $s=x \omega_{0} / c-$ безразмерная координата, $\varphi$ - безразмерная компонента вектор-потенциала, $\omega_{0}$ - плазменная частота в ГСР, $b$ - параметр, который зависит от конфигурации сверхрешетки (период, поверхностная концентрация носителей заряда, тип материала подложки). Уравнение (1) получено для сверхрешетки, представляющей собой 
лист графена, помещенный на подложку, состоящую из чередующихся полосок различных диэлектриков. Отметим, что при $b \ll 1$ уравнение (1) переходит в известное уравнение SGE.

Кинковое решение уравнения (1) задается в неявном виде интегральным соотношением [10]

$$
\int_{\pi}^{\varphi} \frac{d \alpha}{\sqrt{\sqrt{1+b^{2}(1-\cos \alpha)}-1}}=2 \chi
$$

где $\chi=(s-\beta \tau) / \sqrt{1-\beta^{2}}, \beta=v / c, v-$ скорость ЭМ волны в среде, $c$ - скорость света в вакууме. Возникает естественный вопрос - является ли это решение солитонным? В частности, как изменяется форма сталкивающихся уединенных волн после их соударения? Известно, что бегущие навстречу солитоны и/или антисолитоны проходят друг сквозь друга, как полностью проницаемые, и единственный наблюдаемый эффект - фазовый сдвиг. При этом сталкивающиеся солитоны сохраняют свою скорость и форму.

\section{Численный анализ эволюции формы кинка}

Исследуем численно столкновение двух кинков уравнения (1). Интересуемся решениями с $b>1$.

Так как начальное условие в виде (2) задается неявно, уже в начальной форме кинка могут быть существенные
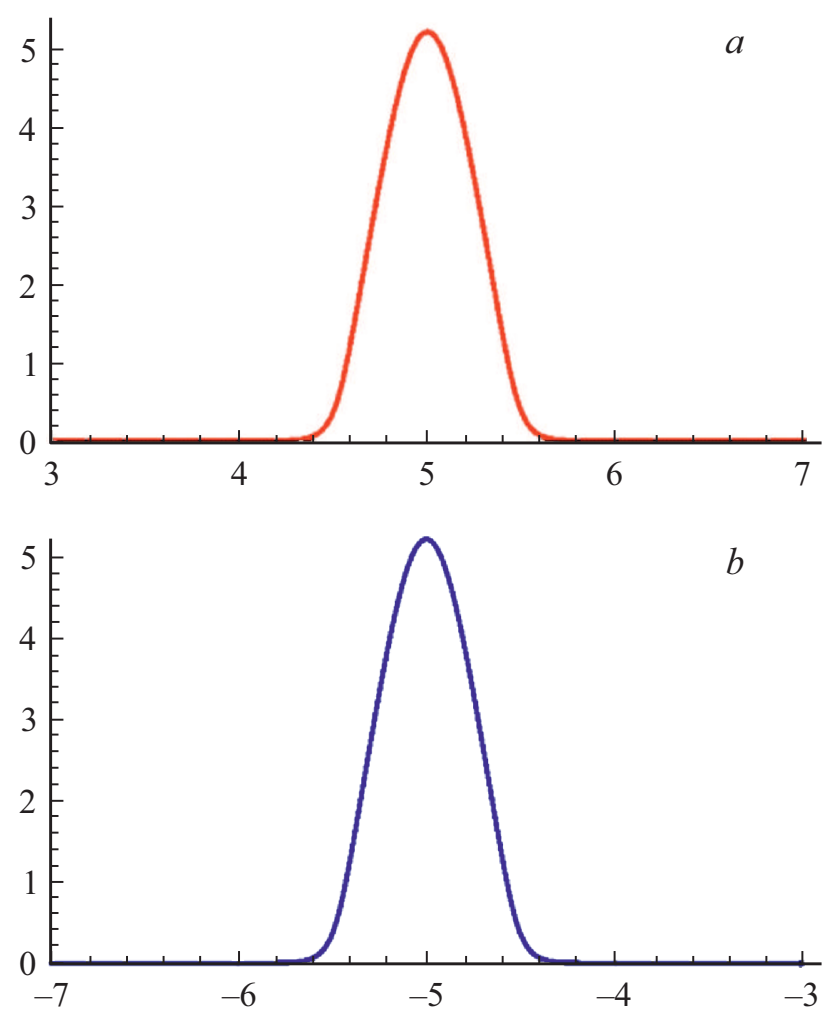

Рис. 1. Форма одиночного кинка в моменты времени $0(a)$ и $20(b)$.
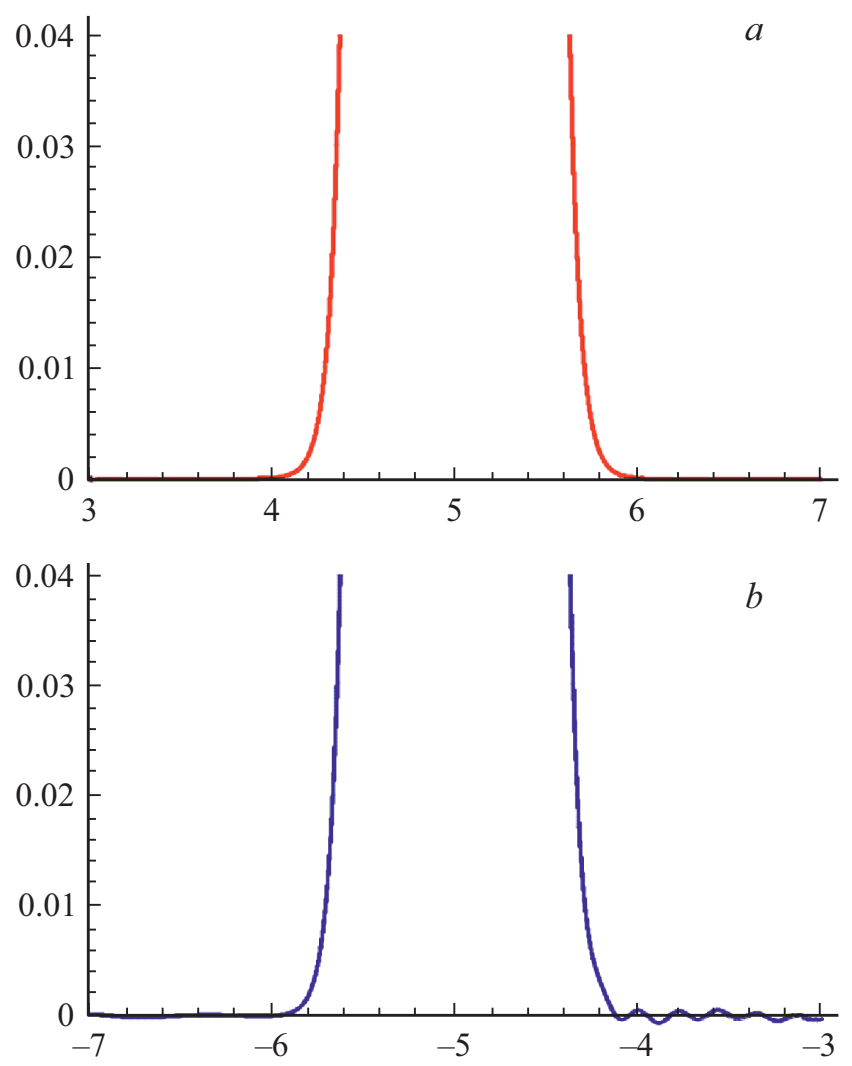

Рис. 2. Форма одиночного кинка в моменты времени $0(a)$ и $20(b)$ при увеличении масштаба.

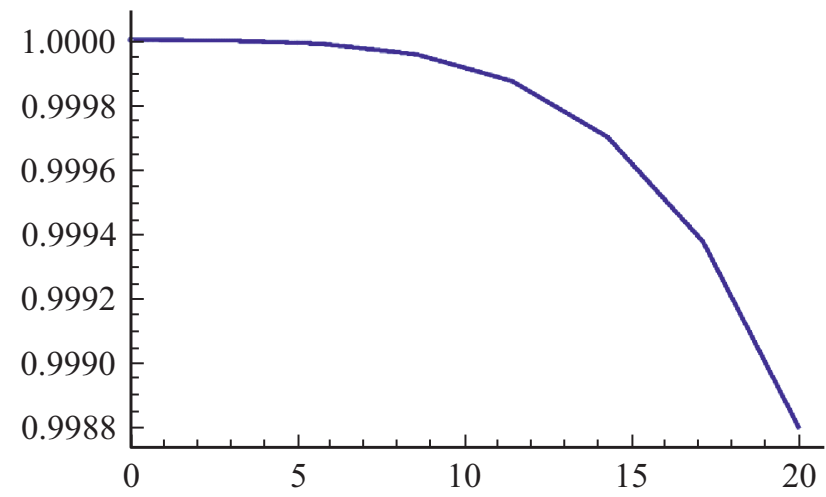

Рис. 3. Зависимость коэффициента корреляции между формой одиночного кинка в начальный момент времени и формой кинка в текущий момент времени.

вычислительные ошибки, обусловленные необходимостью решения интегрального уравнения. Эти ошибки будут накапливаться, влиять на качество решения, и поэтому нам нужен какой-то критерий, позволяющий определить, чем (и насколько) обусловлено изменение формы импульса - вычислительными погрешностями или природой уравнения. В качестве такого критерия возьмем отличие от единицы коэффициента корреляции двух векторов а и $\mathbf{b}$ одинаковой длины $n$, определяемого 
как

$$
\operatorname{corr}(\mathbf{a}, \mathbf{b})=\sum_{i=1}^{n} \frac{\left(a_{i}-\bar{a}\right)\left(b_{i}-\bar{b}\right)}{(n-1)^{2} \operatorname{std}(\mathbf{a}) \operatorname{std}(\mathbf{b})} .
$$

Здесь $\operatorname{std}(\mathbf{x})$ - стандартное отклонение от среднего для вектора $\mathbf{x}$.

Из определения (3) следует, что в случае полного совпадения векторов $\mathbf{a}$ и $\mathbf{b}$ коэффициент $\operatorname{corr}(\mathbf{a}, \mathbf{b})=1$. И чем больше один вектор отличается от другого, тем меньше будет $\operatorname{corr}(\mathbf{a}, \mathbf{b})$.

В качестве векторов а и b будем брать равномерно распределенные массивы значений безразмерной напряженности поля импульса $\partial \varphi / \partial x$ между точками, взятыми на одинаковом расстоянии влево и вправо от вершины импульсов в начальный (вектор а) и какой-либо произвольный (вектор b) моменты времени.

Решение уравнения (1) проводилось с помощью среды „Wolfram Mathematica 10“ [16]. Начальное условие интерполировалось многочленом второй степени из набора точек, полученных решением уравнения (2), с шагом по $\chi=10^{-5}$.

Для оценки вычислительной ошибки, вносимой в начальном условии неявным заданием формы кинка, рассмотрим распространение одиночного кинка в уравнении, сильно отклоняющемся от уравнения sine-Gordon, т.е. $b \gg 1$. Для примера $b=15, v=0.5$, начальная координата $x_{0}=5$. На рис. 1 показаны его положения в моменты времени 0 и 20. На рисунке не видно никаких изменений формы импульса с течением времени. Но при
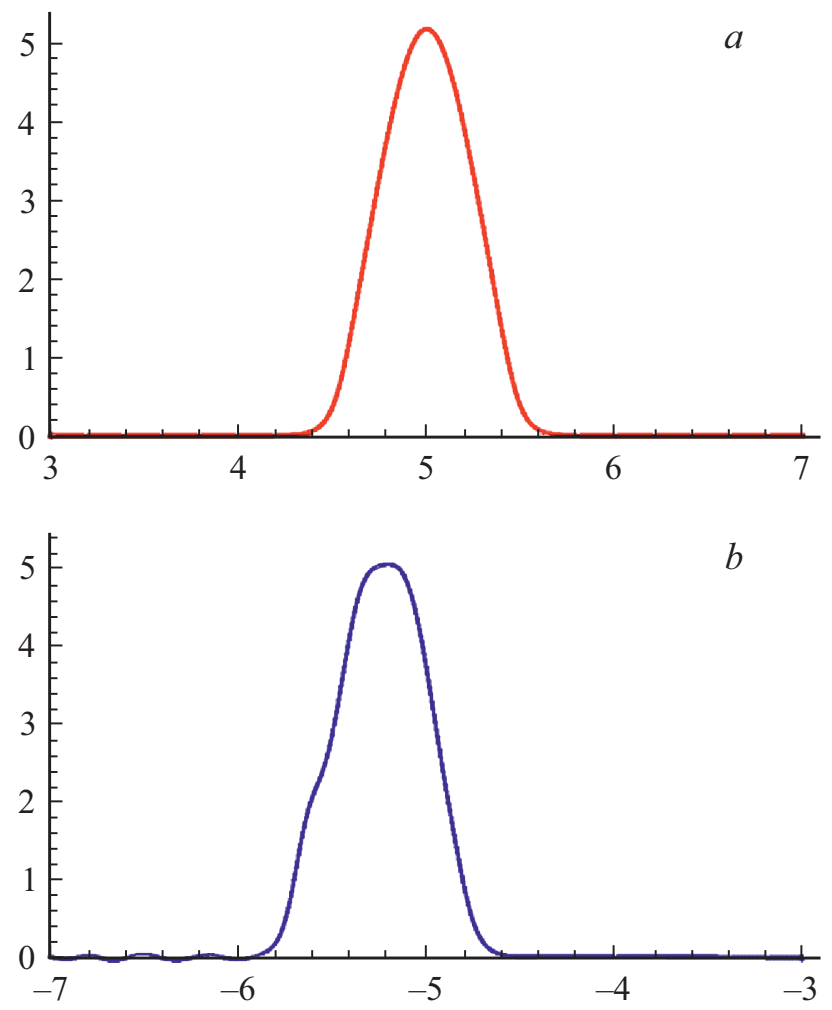

Рис. 4. Форма одного из сталкивающихся кинков в моменты времени $0(a)$ и $20(b)$.
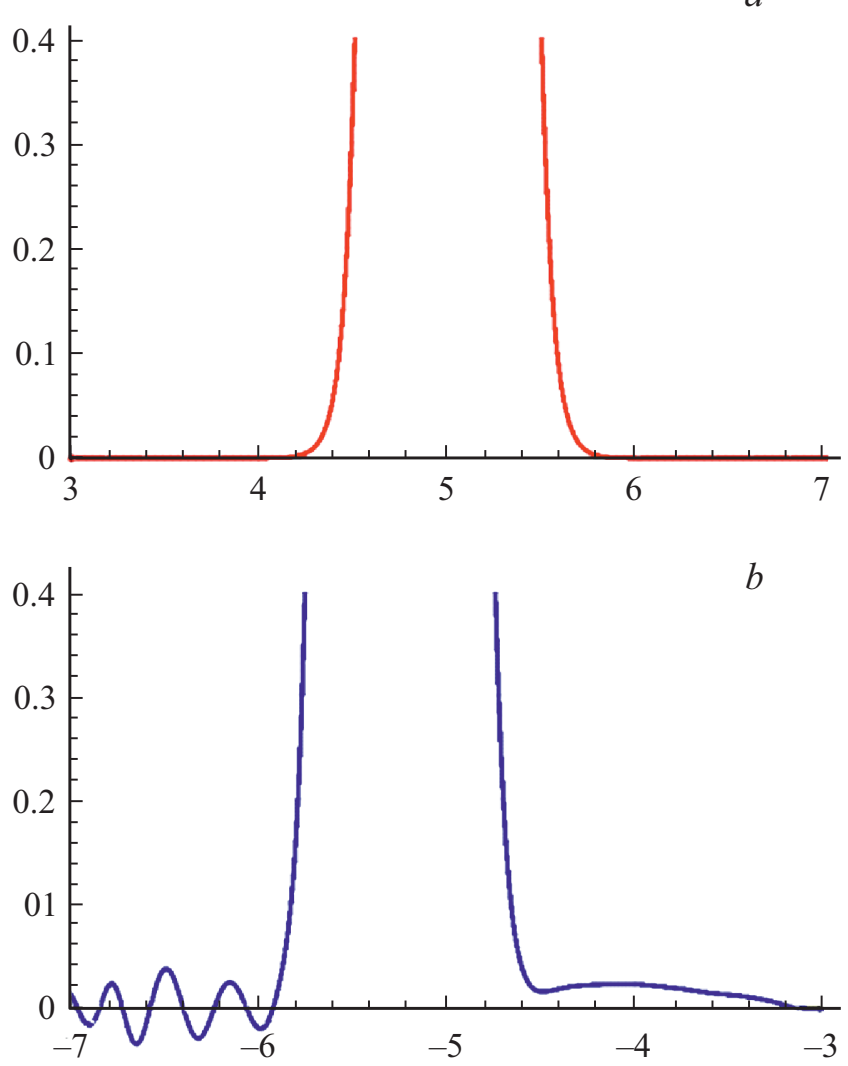

Рис. 5. Форма одного из сталкивающихся кинков в моменты времени $0(a)$ и $20(b)$ при увеличении масштаба.

увеличении (рис. 2) становится заметно, что есть некоторый шум, который усиливается с течением времени.

Этот шум является вычислительной ошибкой и приводит к изменению начальной формы импульса. Для оценки этого изменения найдем коэффициент корреляции (3) формы импульса в разные моменты времени (рис. 3).

Видно, что на времени до 20 единиц изменения есть только в четвертом знаке после запятой. Такой порядок изменений является отправной точкой при исследовании изменения формы импульсов при их столкновении.

Рассмотрим далее столкновение импульсов. Характеристики уравнения и импульсов прежние.

На рис. 4 форма одного из сталкивающихся импульсов показана в моменты времени 0 и 20. Здесь уже невооруженным взглядом заметно изменение формы импульса. Видно, что после столкновения амплитуда кинков стала заметно меньше, чем вначале. Приведем также укрупненный профиль импульсов на рис. 5.

Видно, что амплитуда шума минимум на порядок превосходит амплитуду шума, обусловленную только вычислительными ошибками, т. е. даже визуальный анализ приводит к выводу о том, что кинки уравнения (1), повидимому, не являются солитонами. Этот вывод также подтверждает и расчет коррелятора по формуле (3) в на- 
чальный момент времени и далеко после столкновения

$$
\operatorname{corr}(\tau=0)=1, \quad \operatorname{corr}(\tau=20)=0.91 .
$$

Сравнение $\operatorname{corr}(\tau=20)=0.912139$ в случае столкновения кинков и $\operatorname{corr}(\tau=20)=0.99986$ (рис. 3) в случае распространения одиночного импульса подтверждает вывод о том, что форма импульса в случае столкновения Двух кинков меняется гораздо существенней, чем в случае распространения одиночного импульса.

Таким образом, на основании численного исследования процесса столкновения двух кинков уравнения (1) можно сделать вывод о том, что это уравнение, скорее всего, не имеет солитонных решений.

\section{Финансирование работы}

Работа выполнена при финансовой поддержке Министерства образования и науки РФ на выполнение государственных работ в сфере научной деятельности в рамках проектной части государственного задания, код проекта 3.2797.2017/4.6.

\section{Конфликт интересов}

Авторы заявляют, что у них нет конфликта интересов.

\section{Список литературы}

[1] Екомасов Е.Г., Гумеров А.М., Кудрявщев Р.В. // Письма в ЖЭТФ. 2015. Т. 101. Вып. 12. С. 935939. DOI: 10.7868/S0370274X15120127. [Ekomasov E.G., Gumerov A.M., Kudryavtsev R.V. // JETP Lett. 2015. Vol. 101. N 12. P. $835-839$. https://doi.org/10.1134/S0021364015120061]

[2] Geng X., Shen J., Xue B. // Wave Motion. 2018. Vol. 79. P. 44-56. DOI: 10.1016/j.wavemoti.2018.02.009

[3] Гумеров А.М., Екомасов Е.Г., Муртазин Р.Р., Назаров В.Н. // Журн. вычислит. матем. и матем. физ. 2015. Т. 55. Вып. 4. С. 631-640. DOI: 10.7868/S0044466915040031 [Gumerov A.M., Ekomasov E.G., Murtazin R.R., Nazarov V.N. // Comp. Math. Math. Phys. 2015. Vol. 55. P. 628-637. DOI: $10.1134 / \mathrm{S} 096554251504003 \mathrm{X}]$

[4] Gonzalez J.A., Bellorin A., Garcia-Nustes M.A., Guerrero L.E., Jimenez S., Vazquez L. // Phys. Lett. A. 2017. Vol. 381. N 24. P. 1995-1998.

DOI: 10.1016/j.physleta.2017.03.042

[5] Hua-Zhu L., Shi-Yu L., Ming-Zhu S. // Chin. Phys. B. 2013. Vol. 22. N 4. P. 047807. DOI: $10.1088 / 1674-1056 / 22 / 4 / 047807$

[6] Wang Z., Wang B., Wang K., Long H., Lu P. // Opt. Lett. 2016. Vol. 41. N 15. P. 3619-3622. DOI: $10.1364 /$ OL.41.003619

[7] Adamashvili G.T., Kaup D.J. // Phys. Rev. A. 2017. Vol. 95. P. 053801. DOI: 10.1103/PhysRevA.95.053801

[8] Dong H., Conti C., Marini A., Biancalana F. // J. Phys. B-At. Mol. Opt. 2013. Vol. 46. P. 155401. DOI: $10.1088 / 0953-4075 / 46 / 15 / 155401$
[9] Cuevas-Maraver J., Kevrekidis P.G., Williams F. The sineGordon model and its applications: from pendula and Josephson junctions to gravity and high-energy physics. Springer, 2014. $263 \mathrm{p}$.

[10] Kryuchkov S.V., Kukhar' E.I. // Physica B. 2013. Vol. 408. P. 188-192. DOI: 10.1016/j.physb.2012.09.052

[11] Martin-Vergara F., Rus F., Villatoro F.R. Solitary waves on graphene superlattices, In: Archilla J., Palmero F., Lemos M., Sánchez-Rey B., Casado-Pascual J. (Eds.). Nonlinear Systems, Vol. 2. Understanding Complex Systems. Cham, Springer. P. 85-110.

[12] Kryuchkov S.V., Kukhar' E.I. // Physica E. 2013. Vol. 48. P. 96-100. DOI: 10.1016/j.physe.2012.12.004

[13] Kryuchkov S.V., Kukhar' E.I., Zav'yalov D.V. // Laser Phys. 2013. Vol. 23. P. 065902. DOI: $10.1088 / 1054-660 \mathrm{X} / 23 / 6 / 065902$

[14] Крючков С.В., Кухарь Е.И. // Опт. и спектр. 2015. Т. 118. Вып. 1. C. 163-168. DOI: 10.7868/S0030403415010146 [Kryuchkov S.V., Kukhar' E.I. // Opt. Spectr. 2015. Vol. 118. N 1. P. 157-162. DOI: 10.1134/S0030400X15010142]

[15] Kryuchkov S.V., Kukhar E.I. // Chaos. 2015. Vol. 25. N 7. P. 073116. DOI: $10.1063 / 1.4926944$

[16] Wolfram Mathematica. http://www.wolfram.com/mathematica/, 2018 (accessed 25 September, 2018). 